\title{
Adherence to dosing schedule of denosumab therapy for osteoporosis during COVID-19 lockdown: an electronic medical record and pharmacy claims database study from Asia
}

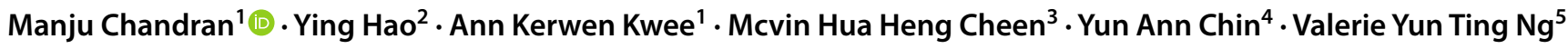

Received: 22 March 2021 / Accepted: 26 July 2021 / Published online: 21 August 2021

(c) International Osteoporosis Foundation and National Osteoporosis Foundation 2021

\begin{abstract}
Summary COVID-19 lockdowns have impacted management of chronic diseases such as osteoporosis. Adherence to the 6-monthly dosing schedule of denosumab, the parenteral anti-osteoporosis medication most often used in Singapore, was significantly reduced during the lockdown period compared to that during pre-COVID-19 times. Patients managed by endocrinologists were more likely to be adherent.

Purpose No study thus far has quantified actual adherence rates to anti-osteoporosis therapy with denosumab during COVID19 or explored factors associated with it. We aimed to estimate the adherence rates to denosumab in Singaporean men and women during COVID-19 lockdown and to compare it with those during the pre-COVID-19 period.

Method We conducted this retrospective, electronic medical records, and pharmacy claims database study at Singapore General Hospital, the largest hospital in the country. Patients initiated on subcutaneous denosumab between August 2019 and December 2019 and were thus scheduled to receive the second dose during the COVID-19 first-wave period from February 2020 to June 2020 (lockdown group) were analyzed, as were patients initiated anytime on denosumab between September 2011 and December 2018 (pre-COVID-19 group). Data extracted from the hospital's electronic prescription platform and patients' pharmacy purchase records were matched. Adherence was defined as being punctual (with an allowable delay of up to 4 weeks) with the second dose scheduled 6 months from the 1st dose. A sensitivity analysis with an allowable delay up to 8 weeks was also performed. We compared the adherence rates between the two periods and explored factors associated with adherence.

Results A total of 768 and 1458 patients respectively during the lockdown and pre-COVID-19 periods were analyzed. The mean adherence rate during lockdown was $63.9 \%$. The odds of being adherent during lockdown were higher if patients were managed by endocrinologists as opposed to those by other specialists [OR 2.516 (95\% CI: 1.836-3.448); $p<0.001$ ]. Adherence rates during the pre-COVID-19 period was $75.4 \%$. Overall, the odds of being adherent to denosumab was significantly lower during lockdown than that during the pre-COVID-19 period [OR 0.525 (95\% CI 0.430-0.640); $p<0.001$ ], and odds of being adherent were higher if patients were managed by endocrinologists than if they were managed by other specialists (OR 1.765 (95\% CI: $1.444-2.158 ; p<0.001$ ).

Conclusion Adherence to denosumab was significantly lower during COVID-19 lockdown than the pre-COVID-19 period. The odds of being adherent were higher in patients managed by endocrinologists. Whether healthcare providers from certain specialties spend more time counselling and educating patients about the importance of adherence to osteoporosis medications needs to be explored further.
\end{abstract}

Keywords COVID-19 $\cdot$ Adherence $\cdot$ Osteoporosis $\cdot$ Parenteral $\cdot$ Denosumab $\cdot$ Compliance

Ann Kerwen Kwee, Mcvin Hua Heng Cheen, and Yun Ann Chin contributed equally.

Manju Chandran

manju.chandran@singhealth.com.sg

Extended author information available on the last page of the article

\section{Introduction}

Osteoporosis and its complication of fragility fractures place a huge health economic burden on societies [1]. Though several medications are currently available for osteoporosis treatment, poor adherence to such therapy is often 
seen. Non-adherence limits the benefits of therapy and is of potential economic significance and international osteoporosis guidelines emphasize the importance of adherence to anti-osteoporosis therapy [2]. Denosumab is a human monoclonal antibody that is approved for the treatment of both post-menopausal and male osteoporosis. It is administered as a $60-\mathrm{mg}$ dose through a subcutaneous injection every 6 months. Denosumab was approved for the treatment of post-menopausal osteoporosis in Singapore in 2011 and for the treatment of male osteoporosis in 2014. Its use in Singapore has increased significantly in the last 2 years, and it is the most often prescribed injectable therapy for osteoporosis in the country currently. Intravenous zoledronic acid and subcutaneous daily teriparatide are much less frequently used $[1,3]$.

Delayed denosumab injections have been shown to be associated with suboptimal BMD response at both spine and total hip [4]. Concerns also exist about loss of gained bone mineral density and rebound vertebral fractures associated with missing denosumab doses or with delay in dosing schedules [5]. This increased risk of vertebral fractures is reported as early as $4-8$ weeks after interruptions in injection schedule [6], and therefore, it is recommended that injections not be delayed by more than 7 months after the last dose [7].

In Singapore, denosumab is administered in clinics at the hospitals and in private specialist and general practitioner clinics by nurses or physicians. This is because of the risk (albeit very rare: 5 reported cases in the post-marketing setting $[8,9]$ of anaphylactic reaction with denosumab. The largest volume of denosumab injections is given in the specialist outpatient clinics at the public restructured hospitals. It is neither available nor dispensed in the "neighborhood" government polyclinics.

COVID-19 has almost completely dominated healthcare priorities and delivery all over the world in the last one and a quarter years. This has noticeably impeded the ability of health systems to deal with other chronic diseases such as osteoporosis. Singapore recorded its first COVID-19 case on 23 January 2020, and, on 7 February 2020; the Disease Outbreak Response System Condition (DORSCON) level was raised from Yellow to Orange in the country. The 2020 Singapore "circuit breaker (CB)" was a stay-at-home order implemented as a preventive measure by the Government of Singapore [10-12]. This was equivalent to the "lockdown" in other countries. During the above period which lasted until 2 June 2020, though all public and private acute hospitals and neighborhood polyclinics remained open for the delivery of essential services, all non-essential appointments were ordered to be deferred, and any on-site staffing was kept to a minimum [13] in order for hospitals and healthcare facilities to accommodate the potential onslaught of COVID-19 patients.
Some survey-based studies have noted that COVID-19 has had a negative impact on osteoporosis care $[14,15]$. We too observed that during the lockdown period in Singapore, patients seemed to be hesitant to come to the hospitals and clinics for ongoing management of their chronic medical conditions including osteoporosis. A study conducted in Austria reported a drop in filled prescriptions for parenteral osteoporosis therapies during the country's lockdown period [16]. From this observed drop, the authors surmised that adherence to such parenteral therapies decreased during lockdown [16]. No attempts however have been made so far anywhere in the world to evaluate the actual adherence rates (as defined conventionally $[17,18]$ ) to parenteral therapies for any disease condition including osteoporosis before and during lockdown periods and to assess the factors associated with the adherence.

Recent reports indicate worsened outcomes of patients with hip fractures and COVID-19 infections, with COVID19 independently associated with 30-day mortality risk after hip fracture [19]. We were concerned that interruption of osteoporosis treatment could have grave implications on the health of our patients. This prompted us to assess the adherence rates of male and female patients with osteoporosis to denosumab (Prolia ${ }^{\circledR}$ ) during the lockdown (circuit breaker) period in Singapore. We conducted the study at Singapore General Hospital (SGH), the largest public hospital in Singapore and among the top ten of the world's largest hospitals [20]. Factors associated with adherence were also assessed. As a secondary aim, we decided to compare the above adherence rates with the adherence of our patients to denosumab injections before the COVID-19 pandemic (pre-COVID-19 adherence). We chose to study denosumab because as mentioned earlier, it is the most often prescribed parenteral therapy for osteoporosis in Singapore and because, unlike as with agents such as IV zoledronic acid, the concern of rebound bone loss and fracture risk exists with interruptions in dosing schedules and/or discontinuation of denosumab.

\section{Methodology}

SGH sees the largest volume of patients with osteoporosis and osteoporotic fragility fractures in the country, and thereby was an appropriate setting for this study. At the clinics in the hospital, prescriptions for denosumab are entered by doctors in the patient prescription system Sunrise Clinical Manager (SCM). The corresponding pharmacy patient records management system - MAXCARE, captures all dispensations. The injection is given on the same day as the purchase is made in the pharmacy (within $30 \mathrm{~min}$ ), and hence, it is understood that if the medicine is bought, it would be administered. 
Ours was an electronic medical records and pharmacy claims database study achieved through a retrospective review of prescription data extracted from SCM and patients' purchase records from MAXCARE. A matching was then done of the actual purchase data against the prescriptions. Since there is a strong likelihood that the medication-taking behavior would be altered if patients had received multiple injections prior, subjects studied in the lockdown group were patients who had been newly initiated on denosumab for the treatment of osteoporosis in the period between August 2019 and December 2019 (index dose). This ensured that there was no overlap with patients started on denosumab earlier and who would have received multiple injections already by then. These patients had been scheduled to receive the second dose in the period February 2020 to June 2020 (the latter time frame being the period right after COVID-19 was identified in Singapore, and restraints at the hospitals and subsequent nation-wide lockdown were instituted). In the pre-COVID-19 group, the same prescription and pharmacy claims data bases were reviewed for patients newly initiated on denosumab between September 2011 and December 2018. As in the lockdown group, the index dose was the first dose that the patient ever received of denosumab during this period, and we examined whether the patient came back for the second dose of the medication 6 months later.

Though not without limitations, adherence assessment from pharmacy administrative data is convenient, noninvasive, and objective. Adherence from such data bases can be measured through several ways. Proportion of days covered (PDC) and compliance to dosing schedule are examples of measures of adherence using refill records. PDC is calculated as the total number of days covered by the medication, divided by the number of days in the period for the individual patient [17]. In the case of denosumab, PDC may not be the most appropriate measure of adherence since the implications of missing denosumab doses might differ depending on whether the number of days missed are simultaneously in large blocks or whether they are consecutively in smaller blocks (e.g., a patient who is on time for one injection but delays the next one by 2 months will have the same PDC as someone who delays both injections by 1 month each, but the two patients will likely will have differing risks of rebound bone loss and fractures). Measuring adherence to dosing schedule might be thus more clinically appropriate [18]. Therefore, we chose this latter method of measuring adherence, and it was defined as receiving the second denosumab injection on time, i.e., at 6 months (with an allowable delay of another 4 weeks) after the date of the index dispensation. We decided to give only a 4-week allowance (grace period) because most previous adherence studies for osteoporosis treatments (oral or otherwise) have used similar windows of allowable delay [18, 21]. International osteoporosis bodies have also recommended that denosumab injections not be delayed beyond 4 weeks from when it is due [7]. In addition, we had also anecdotally been observing in our practice that patients were presenting with fractures after just a month of missing denosumab doses. Pre-COVID-19 adherence was defined as filling the prescription and receiving the second dose of denosumab 6 months from the first dose with an allowable delay of 4 weeks (to match the analysis performed during the lockdown period and to allow comparison with it). Non-adherence to a dose was taken if the dispensed date exceeded 4 weeks from the expected dispensed date. To see if permitting a further allowance of another 4 weeks would result in a higher rate of adherence, a sensitivity analysis with an 8-week allowable delay was performed in the lockdown and pre-COVID-19 groups.

Individuals who were prescribed denosumab but were subsequently taken off it by the prescribing physician due to intolerance or side effects from the medication were not included in the analysis. Patients who were overseas medical tourists were also excluded (Fig. 1a and b).

Considering an anticipated proportion of patients who are adherent to dosing schedule ( 0.60 in the lockdown group and 0.75 in the pre-COVID- 19 group, desired precision $=0.05$; and a confidence level $=0.95$ ), sample sizes of 251 and 126 were respectively required.

Factors that may play a role in adherence/non-adherence like age, sex, ethnicity, fracture history, financial class, and specialty under which patient was being treated for osteoporosis were assessed. Clinical data collected from review of electronic medical records included information on preexisting comorbid diseases categorized under the Charlson Comorbidity Index (CCI) for each patient.

The index applies to 17 disease categories, the scores of which are added to obtain an overall score for each patient [22].

Approval for the study was obtained from the Centralized Institutional Review Board of our institution, with waiver of consent approval also obtained (CIRB Ref 2020/2557). The study conforms to the provisions of the World Medical Association Declaration of Helsinki.

\section{Statistical analyses}

Patient demographics (age, sex, and ethnicity) and other characteristics (financial class, specialty under which patient was managed, fracture history, and adherence) were summarized using frequencies (percentages) for categorical parameters and mean (SD) for continuous variables. To investigate the association of socio-demographic and clinical covariates with adherence, we performed 2-sample $t$-test for continuous variables and chi-square test for categorical variables. Multivariable (MV) logistic regression adjusted for covariates was performed to assess the factors associated with adherence in the lockdown and pre-COVID-19 periods, and to 


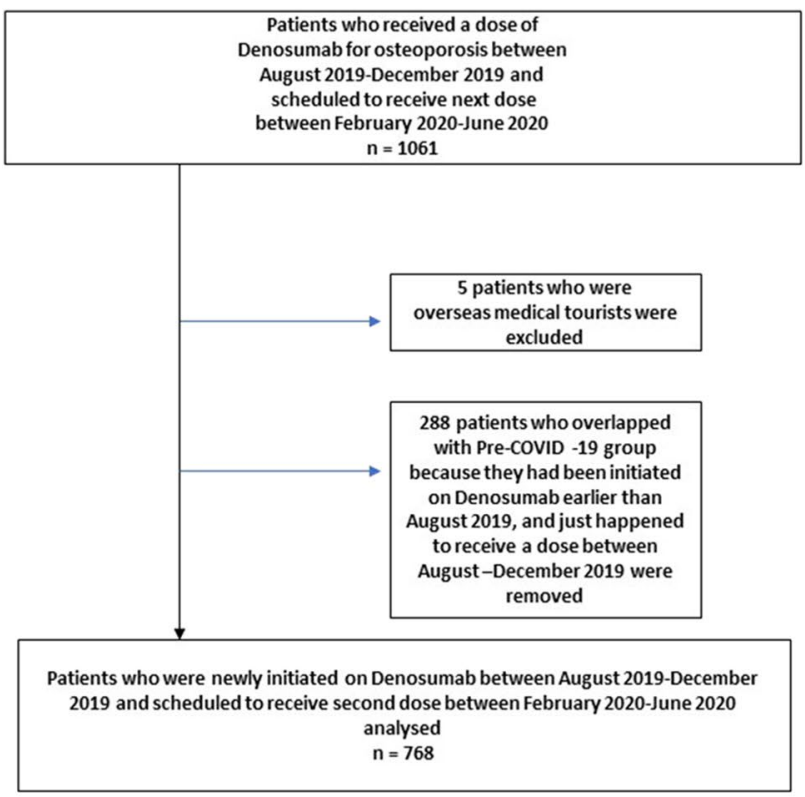

a

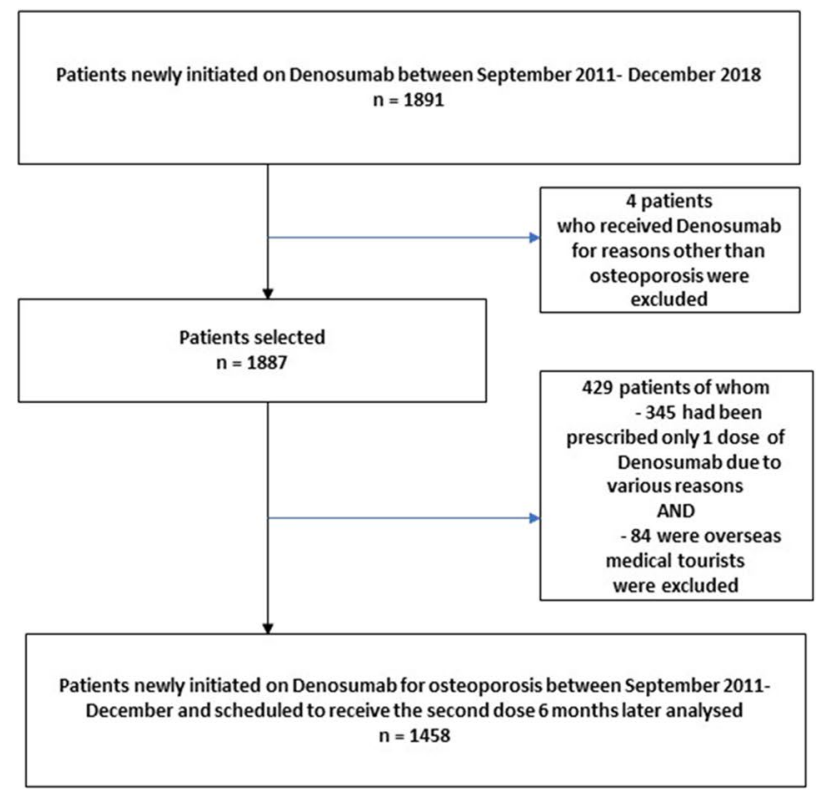

b

Fig. 1 The a Flow diagram for subjects in the lockdown period. b Flow diagram for subjects in the the pre-COVID-19 period

assess factors associated with overall adherence. All tests of significance were two-tailed with significant level of 0.05 .

The analysis was performed using IBM® SPSS Statistics 20.0. Results were taken as statistically significant if the corresponding $p$ value was below 0.05 .

\section{Results}

Lockdown group A total of 768 patients newly initiated on denosumab during the study period (August 2019-December 2019) were analyzed. The study population flow diagram is shown in Fig. 1a.

Descriptive statistics of the lockdown group are summarized in Table 1. The mean (SD) age was $76.0( \pm 9.3)$, and 695 of the 768 patients were women. Mean CCI was $3.8( \pm 1.4)$. Chinese constituted the majority (94.7\%). In total, $84.5 \%$ were patients who belonged to the subsidized financial category (i.e., receiving subsidy benefits for consultation, investigations, and medications). In total, $60.8 \%$ were patients who were managed by endocrinologists; the remainder were managed by departments such as orthopedics, rheumatology, internal medicine, and family medicine. Median time interval between the 1 st and 2 nd dose was 189 days (IQR: 179-241.75).

In total, $63.9 \%$ of patients were found to be adherent to the dosing schedule during the lockdown period. Adherence significantly differed between patients managed by endocrinologists as opposed to those managed by other specialists $(p<0.001)$ (Table 1). There were no significant differences in age, sex, ethnicity, CCI, or financial class between adherent and non-adherent patients (Table 1). Neither was any significant difference noted in adherence between patients with or without a history of fragility fractures.

On multivariate logistic regression analysis, after adjusting for covariates, the odds of being adherent were higher if patients were managed by endocrinologists (Specialty: $p<0.001$, endocrinology versus others: OR 2.516 (95\% CI: 1.836-3.448)] (Table 2).

In the sensitivity analysis with the 8-week allowance, $73.5 \%$ of patients were found to be adherent. As was seen in the primary analysis (6 months with an allowable delay of 4 weeks), the only significant difference noted between adherent and non-adherent patients in the sensitivity analysis was specialty under which the patient was managed, with patients managed by endocrinologists found to be more adherent when compared to those who were managed by other specialists $(p<0.001)$ (Table 3$)$. This remained significant on the multivariate logistic regression analysis (specialty; $p<0.001$, endocrinology vs others: OR 2.096 (95\% CI: 1.497-2.933).

Pre-COVID-19 group A total of 1458 patients were included in the final analysis. The study population flow diagram is shown in Fig. 1b. In the primary analysis of the preCOVID-19 group, $75.4 \%$ of patients were found to be 
Table. 1 Demographics and clinical characteristics of lockdown group

\begin{tabular}{|c|c|c|c|c|}
\hline Characteristic & $N=768$ & Adherent $(N=491)$ & Non-adherent $(N=277)$ & $p$-value \\
\hline Age, years, mean (SD) & $76.0(9.3)$ & $76.3(9.2)$ & $75.6(9.4)$ & 0.358 \\
\hline Charlson Comorbidity Index (CCI), mean (SD) & $3.8(1.4)$ & $3.8(1.5)$ & $3.7(1.3)$ & 0.182 \\
\hline Time interval from previous dose, mean (SD) & $219.7(66.7)$ & $181.9(14.0)$ & 286.5 (70.7) & $<0.001$ \\
\hline Sex, number $(\%)$ & & & & 0.347 \\
\hline Male & $73(9.5)$ & $43(58.9)$ & $30(41.1)$ & \\
\hline Female & $695(90.5)$ & $448(64.5)$ & $247(35.5)$ & \\
\hline Ethnicity, number (\%) & & & & 0.699 \\
\hline Chinese & 729 (94.9) & $469(64.3)$ & $260(35.7)$ & \\
\hline Malay & $6(0.8)$ & $4(66.7)$ & $2(33.3)$ & \\
\hline Indian & $25(3.3)$ & $14(56.0)$ & $11(44.0)$ & \\
\hline Eurasian, etc & $8(1.0)$ & $4(50.0)$ & $4(50.0)$ & \\
\hline Medical specialty, number (\%) & & & & $<0.001$ \\
\hline Other specialties & $301(39.2)$ & $156(51.8)$ & $145(48.2)$ & \\
\hline Endocrinology & $467(60.8)$ & 335 (71.7) & $132(28.3)$ & \\
\hline Financial class, number $(\%)$ & & & & 0.823 \\
\hline Subsidized & $649(84.5)$ & $416(64.1)$ & $233(35.9)$ & \\
\hline Full-paying & $119(15.5)$ & $75(63.0)$ & $44(37.0)$ & \\
\hline Fracture history, number (\%) & & & & 0.593 \\
\hline No & $754(98.2)$ & $483(64.1)$ & $271(35.9)$ & \\
\hline Yes & $14(1.8)$ & $8(57.1)$ & $6(42.9)$ & \\
\hline
\end{tabular}

Bolded numbers indicate significance

adherent. The median time interval between the 1 st and 2nd dose was 183 days (IQR: 175-202). Descriptive statistics of the pre-COVID-19 group are provided in Table 4. As with the lockdown group, significant differences were noted in adherence rates between patients managed by endocrinologists and those by other specialists (Table 4). On the sensitivity analysis with an 8-week allowable delay in the pre-COVID-19 group, $85.8 \%$ were found to be adherent.
Table. 2 Multivariate logistic regression of factors predicting adherence to denosumab in the lockdown group

\begin{tabular}{lllll}
\hline & Odds ratio & Lower 95\% CI & Upper 95\% CI & $p$-value \\
\hline $\begin{array}{l}\text { Specialty } \\
\text { Other specialties }\end{array}$ & Reference & Reference & Reference & Reference \\
Endocrinology & 2.516 & 1.836 & 3.448 & $<\mathbf{0 . 0 0 1}$ \\
$\begin{array}{l}\text { Financial class } \\
\text { Subsidized }\end{array}$ & Reference & Reference & Reference & Reference \\
Full-paying & 1.114 & 0.730 & 1.700 & 0.616 \\
Sex & & & & Reference \\
Male & Reference & Reference & Reference & 0.341 \\
Female & 0.781 & 0.470 & 1.298 & \\
Ethnicity & & & & Reference \\
Chinese & Reference & Reference & Reference & 0.349 \\
$\quad$ Other races & 0.862 & 0.632 & 1.176 & Reference \\
History of fracture before & initiation of denosumab & & 0.669 \\
$\quad$ No & Reference & Reference & Reference & 0.771 \\
Yes & 1.269 & 0.425 & 3.786 & 0.051 \\
Age & 0.997 & 0.977 & 1.018 & 1.149 \\
Charlson Comorbidity & 1.149 & 0.999 & & \\
Index (CCI) & & & &
\end{tabular}

Bolded numbers indicate significance 
Table. 3 Sensitivity analysis of patients in the lockdown group with adherence defined as 6 months + an allowable 8-week delay

\begin{tabular}{|c|c|c|c|c|}
\hline Characteristic & $N=768$ & Adherent $(N=565)$ & $\begin{array}{l}\text { Non-adherent } \\
(N=203)\end{array}$ & $p$-value \\
\hline Age, years, mean (SD) & $76.0(9.3)$ & $76.1(9.3)$ & $75.9(9.3)$ & 0.838 \\
\hline $\begin{array}{l}\text { Charlson Comorbidity Index } \\
\text { (CCI), mean (SD) }\end{array}$ & $3.8(1.4)$ & $3.8(1.5)$ & $3.7(1.2)$ & 0.250 \\
\hline Sex, number $(\%)$ & & & & 0.486 \\
\hline Male & $73(9.5)$ & $51(69.9)$ & $22(30.1)$ & \\
\hline Female & $695(90.5)$ & $514(74.0)$ & $181(26.0)$ & \\
\hline Ethnicity, number (\%) & & & & 0.323 \\
\hline Chinese & $729(94.9)$ & $538(73.8)$ & $191(26.2)$ & \\
\hline Malay & $6(0.8)$ & $5(83.3)$ & $1(16.7)$ & \\
\hline Indian & $25(3.3)$ & $15(60.0)$ & $10(40.0)$ & \\
\hline Eurasian, etc & $8(1.0)$ & $7(87.5)$ & $1(12.5)$ & \\
\hline Medical specialty, number (\%) & & & & $<0.001$ \\
\hline Other specialties & $301(39.2)$ & $197(65.4)$ & $104(34.6)$ & \\
\hline Endocrinology & $467(60.8)$ & $368(78.8)$ & $99(21.2)$ & \\
\hline Financial class, number (\%) & & & & 0.215 \\
\hline Subsidized & $649(84.5)$ & $483(74.4)$ & $166(25.6)$ & \\
\hline Full-paying & $119(15.5)$ & $82(68.9)$ & $37(31.1)$ & \\
\hline Fracture history, number (\%) & & & & 0.668 \\
\hline No & $754(98.2)$ & $554(73.5)$ & $200(26.5)$ & \\
\hline Yes & $14(1.8)$ & $11(78.6)$ & $3(21.4)$ & \\
\hline
\end{tabular}

Bolded numbers indicate significance
Overall adherence to denosumab When the overall adherence to denosumab was examined with an allowable delay of 4 weeks, after adjusting for other variables, the timeframe was significantly associated with adherence, with the

Table. 4 Demographics and clinical characteristics of the pre-COVID-19 group

\begin{tabular}{|c|c|c|c|c|}
\hline Characteristic & $N=1458$ & Adherent $(N=1099)$ & Non-adherent $(N=359)$ & $p$-value \\
\hline Age, years, mean (SD) & $78.2(9.4)$ & $78.2(9.3)$ & $78.4(9.8)$ & 0.718 \\
\hline Charlson Comorbidity Index (CCI), mean (SD) & $4.0(1.6)$ & $4.0(1.5)$ & $4.1(1.7)$ & 0.271 \\
\hline Time interval from previous dose, mean (SD) & $211.7(127.9)$ & $180.6(10.9)$ & $307.0(232.9)$ & $<0.001$ \\
\hline Sex, number $(\%)$ & & & & 0.164 \\
\hline Male & $128(8.8)$ & $90(70.3)$ & 38 (29.7) & \\
\hline Female & $1330(91.2)$ & 1009 (75.9) & $321(24.1)$ & \\
\hline Ethnicity, number (\%) & & & & 0.212 \\
\hline Chinese & $1343(92.1)$ & $1006(74.9)$ & $337(25.1)$ & \\
\hline Malay & $27(1.9)$ & $24(88.9)$ & $3(11.1)$ & \\
\hline Indian & $52(3.6)$ & $43(82.7)$ & $9(17.3)$ & \\
\hline Eurasian, etc & $36(2.5)$ & $26(72.2)$ & $10(27.8)$ & \\
\hline Medical specialty, number (\%) & & & & 0.005 \\
\hline Other specialties & $821(56.3)$ & $596(72.6)$ & $225(27.4)$ & \\
\hline Endocrinology & $637(43.7)$ & $503(79.0)$ & $134(21.0)$ & \\
\hline Financial class, number (\%) & & & & 0.645 \\
\hline Subsidized & $1079(74.0)$ & $810(75.1)$ & $269(24.9)$ & \\
\hline Full-paying & $379(26.0)$ & $289(76.3)$ & $90(23.7)$ & \\
\hline Fracture history, number (\%) & & & & 0.543 \\
\hline No & $1449(99.4)$ & $1093(75.4)$ & $356(24.6)$ & \\
\hline Yes & $9(0.6)$ & $6(66.7)$ & $3(33.3)$ & \\
\hline
\end{tabular}

Bolded numbers indicate significance 
odds of patients being adherent to denosumab significantly lower during lockdown than in the pre-COVID-19 period, $p<0.001$, OR 0.525 (95\% CI: 0.430-0.640). Specialty under which patients were managed remained significant with the odds of patients being higher in adherence if they were managed by endocrinologists, $p<0.001$, OR 1.765 (95\% CI: 1.444-2.158) (Table 5).

When the overall adherence to denosumab was examined with the 8-week allowable delay in the sensitivity analysis, again, the time frame was found to be significantly associated with adherence, with the odds of patients being adherent to denosumab significantly lower during the lockdown than that in the pre-COVID-19 period, $p<0.001$, OR 0.437 (95\% CI: 0.349-0.548). Specialty under which patients were managed remained significant with the odds of patients being adherent higher if they were managed by endocrinologists, $p<0.001$, OR 1.579 (95\% CI: 1.255-1.987).

\section{Discussion}

Our study is the first to provide real-world quantifiable information on adherence rates to a parenteral therapy for osteoporosis, and factors associated with it during the critical period of the lockdown that was part of the containment efforts for COVID-19 worldwide. We found that the adherence rates as defined by complying to the dosing schedule of 6 months + an allowable delay of 4 weeks to denosumab, the most frequently used parenteral therapy for osteoporosis in Singapore, decreased significantly during the lockdown compared to those during the pre-COVID-19 period. This significant decrease was noted when the allowable delay was extended to 8 weeks too. Whether this decrease in adherence that we observed in Singapore during our lockdown is the case in other countries is not known since there are no prior studies that have explored this characteristic.

We found a significant difference in adherence between patients managed by endocrinologists versus other specialists. The reason behind this is unclear. No selection bias is present in our study since the patients were identified from the electronic medical records and included all patients who were prescribed with denosumab at our hospital during the period of study. In the UK Teriparatide study, it was suggested among other factors that patients may be more inclined to be persistent to teriparatide therapy when prescribed the drug by a specialist [23]. However, we could not find any previous study that corroborated specialty under which patients were managed with adherence to an anti-osteoporosis agent. Since COVID-19 and the lockdowns it resulted in came upon abruptly and affected broad swathes of the healthcare spectrum, there was no time to prepare patients for it. Therefore, it is unlikely that any one specialty had any additional time to arrange for patients to receive their injections according to schedule or to inform their patients to be adherent to medications.
Table. 5 Multivariate logistic regression of factors predicting adherence to denosumab

\begin{tabular}{lllll}
\hline & Odds ratio & Lower 95\% CI & Upper 95\% CI & $p$ value \\
\hline $\begin{array}{c}\text { Specialty } \\
\text { Other specialties }\end{array}$ & Reference & Reference & Reference & Reference \\
Endocrinology & 1.765 & 1.444 & 2.158 & $<\mathbf{0 . 0 0 1}$ \\
Time frame & & & Reference & Reference \\
Pre-COVID-19 & Reference & Reference & 0.640 & $<\mathbf{0 . 0 0 1}$ \\
Lockdown & 0.525 & 0.430 & & Reference \\
Financial class & & & Reference & 0.099 \\
Subsidized & Reference & Reference & 1.552 & Reference \\
Full-paying & 1.222 & 0.963 & & 0.809 \\
Ethnicity & & & Reference \\
Chinese & Reference & Reference & 1.212 & Reference \\
Other races & 1.021 & 0.861 & & 0.092 \\
Sex & & & Reference \\
Male & Reference & Reference & 1.045 & Reference \\
Female & 0.763 & 0.557 & & 0.818 \\
History of fracture before initiation of denosumab & Reference & 0.802 \\
No & Reference & Reference & 2.144 & 0.839 \\
Yes & 0.904 & 0.381 & 1.014 & 1.086 \\
Age & 1.002 & 0.990 & & \\
Charlson Comorbidity & 1.008 & 0.935 & & \\
Index (CCI) & & & &
\end{tabular}

Bolded numbers indicate significance 
Also, this significant difference in adherence depending on specialty under which the patient was managed was noted in both the lockdown and the Pre-COVID-19 groups indicating that a systemic difference was present always, and not just during the lockdown period. We have shown earlier that adherence rates to bisphosphonate therapy were higher in patients enrolled into a fracture liaison service (FLS) at our hospital compared to that in conventionally managed osteoporotic patients [24]. The current study was not of patients selected from the FLS or from any particular specialty and yet we found this difference. Whether or not healthcare providers from certain specialties spend more time counselling patients on proper administration of denosumab and the importance of adherence to it needs to be explored further.

Though there is a commonly held belief that when patients have multiple chronic conditions, adherence to medications decreases, we did not observe this in our study. The presence of comorbidities as assessed by the Charlson Comorbidity Index (CCI) did not significantly differ between adherent and non-adherent patients either in the lockdown group or in the pre-COVID-19 group. We found only one other study in the literature that included a comorbidity index as a covariate in analyzing persistence to denosumab [25]. In that study in which the Wolfe Comorbidity index was included as a covariate in the models from Germany and Austria, the authors found a trend towards an association that did not reach statistical significance between the modified Wolfe Comorbidity index and persistence in Germany but not in Austria. The authors of the study did not provide a plausible explanation for this difference.

Though it can be argued that older patients who may lack mobility and independence may find it more difficult to travel to clinics and medical centers to receive a parenteral therapy for their osteoporosis, we found that adherence to denosumab was not influenced by age. This was similar to what was noted in another study [18]. In other studies that have explored adherence to medications in general, conflicting results have been obtained with some reporting that younger age is associated with lower persistence [26, 27], while others suggest that persistence declines with age [28].

We also did not find a significant difference in adherence rates between men and women in our study. Though no previous study has explored differences in adherence to denosumab between men and women, studies that have explored adherence to osteoporosis therapies in general have reported conflicting findings with men found to be less likely to be adherent [29], while others have found the opposite [30].

Adherence to anti-osteoporosis medications and its association with previous history of fracture have varied among studies $[18,24]$. In our study, the presence of previous fracture did not appear to have any association with adherence either during COVID-19 lockdown or in the pre-COVID-19 period. During the COVID-19 lockdown especially, the likelihood of a patient being more receptive to physician advice to be adherent to prescribed treatment regimens following the traumatic experience of a prior fracture was probably outweighed by the concerns and restraints imposed by the COVID-19 lockdown.

No data on socioeconomic status and its association with osteoporosis medication exists. However, a low socioeconomic status has been associated with non-adherence to oral medications in general [31], and previous quasi-experimental studies have demonstrated decreases in medication adherence when out-of-pocket drug costs are high [32]. A reassuring finding from our study was that financial class was not associated with adherence either in the lockdown or in the pre-COVID-19 group.

Our study has some limitations. Since the study's primary aim was to evaluate the adherence of patients to the first lockdown phase during the ongoing COVID-19 pandemic, it was not designed to evaluate the interactions between adherence and risk of rebound fracture after interruption of denosumab therapy. Though conflicting evidence exists as to how long an injection of denosumab would have to be delayed before fracture risk increases [33], the general recommendation as mentioned previously is that injections not be delayed by more than 7 months after the previous injection [7]. The findings from a preliminary analysis that we subsequently performed showed that, overall, fractures on follow-up were less likely to occur in patients who were adherent to their second dose of denosumab at 6 months (with an allowable delay of 4 weeks). Twenty-eight out of $1590(1.8 \%)$ patients in the adherent group sustained subsequent fractures as opposed to $21 / 615(3.4 \%)$ in the nonadherent group ( $p: 0.025)$. This remained significant after adjusting for other variables, with patients adherent to the above dosing schedule having less odds to sustain a fracture ( $p=0.038$, OR: 0.538 (95\% CI 0.300-0.967). This finding also validated our a priori decision in our primary study to use only a 4-week allowable delay in the estimation of adherence to denosumab.

The compulsory anonymization of subjects to comply with the personal data protection act (PDPA) rules of Singapore precluded us from questioning individual patients the reasons for their non-adherence. The exact reasons thus for the decreased adherence rates during the lockdown, i.e., whether it was because of concerns of acquiring the infection by attending busy clinics and hospitals or whether it was because of other reasons such as whether patients developed intercurrent illnesses including COVID are unknown. Data on whether patients had been on other anti-osteoporosis agents in the past, prior to being initiated on denosumab was not collected. It has been suggested in other studies that a history of use of other osteoporosis medications is predictive of higher persistence to denosumab with the likely reason 
postulated that treatment-experienced patients as opposed to treatment-naïve patients would be more informed and knowledgeable about their disease [18, 25, 34, 35]. It must be noted that this postulate has not been conclusively proven. However, we do not believe that a history of prior anti-osteoporosis medication use would have impacted the short-term adherence of our patients to denosumab during the COVID19 lockdown. The data gathered did not have information on the educational level of patients nor was there any information available on presence or absence of caregiver support. Both these may have an impact on adherence though the fact that we did not find that age had an impact on adherence in our study mitigates the lack of the latter information.

Notwithstanding these limitations, our study has several advantages. The large sample size was a distinct advantage. Using an electronic medical record and pharmacy claims database for the study allowed us to capture the pertinent data in its entirety compared to methods such as conducting interviews and surveys which may be hampered by reporting biases, missing data, and inaccurate recall by patients. All the public hospitals in Singapore, a small city-state, have more-or-less similar modalities of practice; our hospital and its clinics see the largest volume of patients in the country including osteoporosis and is representative of the current practice in Singapore as far as denosumab therapy is concerned. Only a minority of patients with osteoporosis in Singapore get denosumab injections at private general practitioner clinics, and it is very unlikely that patients who are undergoing follow-up at public hospital clinics would have switched to private practitioners (either general or specialist) for their medical care during the pandemic. Therefore, it is very unlikely that we would have underestimated adherence to denosumab. The structure of the healthcare system in Singapore is also such that community pharmacies do not dispense medications without prescriptions from a doctor, and private general practitioners and specialists do not switch chronic medications that are prescribed to a patient by a doctor at a public healthcare institution unless patients experience side effects from them. Therefore, it is unlikely that patients could have obtained an alternate osteoporosis medication from elsewhere.

A follow-up study is planned to elucidate whether the patients who were non-adherent and/or who discontinued denosumab in the last 1 year will return for regular follow-up once pandemic restrictions are eased and the circuit breaker (lockdown) is completely lifted. However, as we found in this study, even when an 8-week allowable delay was given in the sensitivity analysis, only a $10 \%$ increase in adherence rate during the lockdown period was noted. On the multivariate logistic regression analysis with the 8-week allowance, the odds of being adherent in the lockdown group were still lower than in the pre-COVID-19 group. This suggests that so long as lockdown and its constraints last, patients are likely to be unwilling to come back to the hospital clinics to receive their parenteral anti-osteoporosis therapies.

A tangential benefit of our findings which revealed that adherence rates to a parenteral therapy for osteoporosis had decreased during the lockdown period is that it has laid the groundwork for our hospital to review response systems and strategies for better delivery of patient care and medications. Streamlining of appointments for denosumab injections and stocking of denosumab in the clinics so that the latter can provide one-stop service thereby avoiding over-crowding at the pharmacies is one such strategy. To reduce footfall in the busy hospital, and to preserve hospital capacity for acutely ill patients, systems of care for chronic diseases are being rolled out including virtual clinics and video consultations. Safe corridors of passage with minimum number of contact points for patients who are coming to the clinics just to get their six monthly denosumab injections have been conceptualized and implemented. Methods of safe home delivery of certain parenteral medications which patients then can take to their neighborhood private GP for administration are also gaining popularity. An in-hospital audit showed that more than $50 \%$ of total prescriptions of medications at our hospital are now home-delivered as opposed to only $20 \%$ before the pandemic. The challenge will be to embed these innovative methods in the normal hospital workflow so that they can become the new-normal and we can prepare for possible new waves of COVID-19 itself or even for other such disease outbreaks and calamities. We are also exploring fine tuning our existing health-technology platforms that are purpose-built to facilitate communication between the hospital and patients for scheduling appointments, to further enable a two-way exchange between doctors and their patients for education and disease management. These platforms can also be potentially modified to emphasize adherence to medications and can be used to remind patients about timely administration especially of parenteral medications. Our findings of decreased adherence to a parenteral therapy for osteoporosis during COVID-19 lockdown and changes made to the response systems and healthcare delivery strategies were prescient. Singapore is going through a second wave of COVID-19 infections currently, and population movements and activities have been curtailed. Hospitals too have had tighter curbs and restrictions reinstituted.

\section{Conclusion}

Our study shows that adherence rates to denosumab (an anti-osteoporosis agent to which patients have traditionally demonstrated better compliance to when compared to other anti-osteoporosis therapies) decreased during the COVID19 lockdown. This decrease in adherence may have grave implications. Repeated "waves" of COVID-19 and the 
lockdowns they will naturally induce may result in a rise in such non-adherence rates even though they may be shortterm. Urgent steps therefore need to be taken to mitigate the fall-out from such lockdowns. Further research into individual patient reasons for non-adherence during such times needs to be undertaken so that measures to address such concerns can be instituted. Better communication by health providers about the critical need for adherence to an agent such as denosumab and alternate options for delivery and safe administration to patients should be considered so that interruptions in medication dosing schedules do not occur.

Acknowledgements The authors would like to acknowledge Mr Set Kuo Lik and Ms Juanillo Christin Joy Ramos from the Singhealth IHIS eHInts Support team for helping to extract the data from Sunrise Clinical Manager and MAXCARE and for deidentifying it for the purpose of the study.

\section{Declarations}

Conflicts of interest None.

\section{References}

1. Chandran M, Lau TC, Gagnon-Arpin I, Dobrescu A, Li W, Leung MYM et al (2019) The health and economic burden of osteoporotic fractures in Singapore and the potential impact of increasing treatment rates through more pharmacological options. Arch Osteoporos 14(1):114

2. Chandran M, Mitchell PJ, Amphansap T, Bhadada SK, Chadha M, Chan DC et al (2021) Development of the Asia Pacific Consortium on Osteoporosis (APCO) Framework: clinical standards of care for the screening, diagnosis, and management of osteoporosis in the Asia-Pacific region. Osteoporos Int 32(7):1249-1275

3. Chau YT, Nashi N, Law LSC, Goh RKH, Choo SX, Seetharaman SK (2020) Undertreatment of osteoporosis following hip fracture: a retrospective, observational study in Singapore. Arch Osteoporos 15(1):141

4. Lyu H, Zhao SS, Yoshida K, Tedeschi SK, Xu C, Nigwekar SU et al (2020) Delayed denosumab injections and bone mineral density response: an electronic health record-based study. J Clin Endocrinol Metab 105(5):1435-1444

5. Tsourdi E, Zillikens MC, Meier C, Body JJ, Gonzalez Rodriguez E, Anastasilakis AD et al (2020) Fracture risk and management of discontinuation of denosumab therapy: a systematic review and position statement by ECTS. J Clin Endocrinol Metab 106(1):264-281. https://doi.org/10.1210/clinem/dgaa756

6. Anastasilakis AD, Evangelatos G, Makras P, Iliopoulos A (2020) Rebound-associated vertebral fractures may occur in sequential time points following denosumab discontinuation: need for prompt treatment re-initiation. Bone Rep 12:100267

7. https://www.osteoporosis.foundation/news/joint-guidance-covid19-vaccination-and-osteoporosis-management-20210309-1500? fbclid=IwAR1dIPM5QH20Ey5YW1W_Re2y0R18eZx62O99 $\mathrm{NZiKe} 4 \mathrm{cO}-8 \mathrm{AgKgw} 2 \mathrm{~N} 1 x c$ [Internet]. [cited March 15 2021]. Available from: https://www.osteoporosis.foundation/news/jointguidance-covid-19-vaccination-and-osteoporosis-management20210309-1500?fbclid=IwAR1dIPM5QH20Ey5YW1W_Re2y0 R18eZx62O99__NZiKe4cO-8AgKgw2N1xc
8. https://www.hsa.gov.sg/announcements/dear-healthcare-profe ssional-letter/prolia-(denosumab-60-mg)-risk-of-anaphylacticreaction-and-new-contraindication-in-patients-with-clinicallysignificant-hypersensitivity-to-prolia [Internet]. [cited February 18 2021]. Available from: https://www.hsa.gov.sg/announceme nts/dear-healthcare-professional-letter/prolia-(denosumab-60mg)-risk-of-anaphylactic-reaction-and-new-contraindication-inpatients-with-clinically-significant-hypersensitivity-to-prolia

9. Geller M, Wagman R (2014) Ho P et alSAT0479 Early findings from Prolia ${ }^{\circledR}$ post-marketing safety surveillance for atypical femoral fracture, osteonecrosis of the jaw, severe symptomatic hypocalcemia, and anaphylaxis. Ann Rheum Dis 73:766-767

10. https://www.pmo.gov.sg/Newsroom/PM-Lee-Hsien-Loong-onthe-COVID19-situation-in-Singapore-on-3-April-2020 [Internet]. Available from: https://www.pmo.gov.sg/Newsroom/ PM-Lee-Hsien-Loong-on-the-COVID19-situation-in-Singa pore-on-3-April-2020

11. https://sso.agc.gov.sg/SL-Supp/S254-2020/Published/20200 407?DocDate $=20200407$ [Internet]. [cited February 18 2021]. Available from: https://sso.agc.gov.sg/SL-Supp/S254-2020/ Published/20200407?DocDate $=20200407$

12. https://www.gov.sg/article/ending-circuit-breaker-phased-appro ach-to-resuming-activities-safely [Internet]. Available from: https://www.gov.sg/article/ending-circuit-breaker-phased-appro ach-to-resuming-activities-safely

13. https://www.moh.gov.sg/news-highlights/details/continuationof-essential-healthcare-services-during-period-of-heightenedsafe-distancing-measures [Internet]. [cited February 18 2020]. Available from: https://www.moh.gov.sg/news-highlights/detai 1s/continuation-of-essential-healthcare-services-during-periodof-heightened-safe-distancing-measures

14. Peeters JJM, van den Berg P, van den Bergh JP, Emmelot-Vonk MH, de Klerk G, Lems WF et al (2021) Osteoporosis care during the COVID-19 pandemic in the Netherlands: a national survey. Arch Osteoporos 16(1):11

15. Fuggle NR, Singer A, Gill C, Patel A, Medeiros A, Mlotek AS et al (2021) How has COVID-19 affected the treatment of osteoporosis? An IOF-NOF-ESCEO global survey. Osteoporos Int 32(4):611-617

16. Kocijan R, Behanova M, Reichardt B, Haschka J, Kocijan A, Zwerina J (2021) Poor adherence to parenteral osteoporosis therapies during COVID-19 pandemic. Arch Osteoporos 16(1):46

17. Cadarette SM, Burden AM (2010) Measuring and improving adherence to osteoporosis pharmacotherapy. Curr Opin Rheumatol 22(4):397-403

18. Silverman SL, Siris E, Belazi D, Recknor C, Papaioannou A, Brown JP et al (2018) Persistence at 24 months with denosumab among postmenopausal women with osteoporosis: results of a prospective cohort study. Arch Osteoporos 13(1):85

19. Hall AJ, Clement ND, Farrow L, MacLullich AMJ, Dall GF, Scott CEH et al (2020) IMPACT-Scot report on COVID-19 and hip fractures. Bone Joint J 102-B(9):1219-28

20. https://www.healthcareglobal.com/top10/top-10-worlds-largesthospitals/singapore-general-hospital-singapore [Internet]. 2021 [cited February 21 2021]. Available from: https://www.healthcare global.com/top10/top-10-worlds-largest-hospitals/singapore-gener al-hospital-singapore

21. Siris ES, Selby PL, Saag KG, Borgström F, Herings RMC, Silverman SL (2009) Impact of osteoporosis treatment adherence on fracture rates in North America and Europe. Am J Med 122(2 Suppl):S3-13

22. Charlson ME, Pompei P, Ales KL, MacKenzie CR (1987) A new method of classifying prognostic comorbidity in longitudinal studies: development and validation. J Chronic Dis 40(5):373-383 
23. Arden NK, Earl S, Fisher DJ, Cooper C, Carruthers S, Goater M (2006) Persistence with teriparatide in patients with osteoporosis: the UK experience. Osteoporos Int 17(11):1626-1629

24. Cheen MHH, Kong MC, Zhang RF, Tee FMH, Chandran M (2012) Adherence to osteoporosis medications amongst Singaporean patients. Osteoporos Int 23(3):1053-1060

25. Fahrleitner-Pammer A, Papaioannou N, Gielen E, FeudjoTepie M, Toffis C, Frieling I et al (2017) Factors associated with high 24-month persistence with denosumab: results of a real-world, non-interventional study of women with postmenopausal osteoporosis in Germany, Austria, Greece, and Belgium. Arch Osteoporos 12(1):58

26. Briesacher BA, Andrade SE, Fouayzi H, Chan KA (2008) Comparison of drug adherence rates among patients with seven different medical conditions. Pharmacotherapy 28(4):437-443

27. van Boven JFM, de Boer PT, Postma MJ, Vegter S (2013) Persistence with osteoporosis medication among newly-treated osteoporotic patients. J Bone Miner Metab 31(5):562-570

28. Lekkerkerker F, Kanis JA, Alsayed N, Bouvenot G, Burlet N, Cahall D et al (2007) Adherence to treatment of osteoporosis: a need for study. Osteoporos Int 18(10):1311-1317

29. Devine J, Trice S, Finney Z, Yarger S, Nwokeji E, Linton A et al (2012) A retrospective analysis of extended-interval dosing and the impact on bisphosphonate compliance in the US Military Health System. Osteoporos Int 23(4):1415-1424

30. Landfeldt E, Ström O, Robbins S, Borgström F (2012) Adherence to treatment of primary osteoporosis and its association to fractures-the Swedish Adherence Register Analysis (SARA). Osteoporos Int 23(2):433-443

31. Wamala S, Merlo J, Bostrom G, Hogstedt C, Agren G (2007) Socioeconomic disadvantage and primary non-adherence with medication in Sweden. Int J Qual Health Care: J Int Soc Qual Health Care 19(3):134-140

32. Goldman DP, Joyce GF, Escarce JJ, Pace JE, Solomon MD, Laouri $M$ et al (2004) Pharmacy benefits and the use of drugs by the chronically ill. JAMA 291(19):2344-2350

33. Lyu H, Yoshida K, Zhao SS, Wei J, Zeng C, Tedeschi SK et al (2020) Delayed denosumab injections and fracture risk among patients with osteoporosis : a population-based cohort study. Ann Intern Med 173(7):516-526

34. Papaioannou A, Khan A, Belanger A, Bensen W, Kendler D, Theoret $\mathrm{F}$ et al (2015) Persistence with denosumab therapy among osteoporotic women in the Canadian patient-support program. Curr Med Res Opin 31(7):1391-1401

35. Tremblay É, Perreault S, Dorais M (2016) Persistence with denosumab and zoledronic acid among older women: a populationbased cohort study. Arch Osteoporos 11(1):30

Publisher's note Springer Nature remains neutral with regard to jurisdictional claims in published maps and institutional affiliations.

\section{Authors and Affiliations}

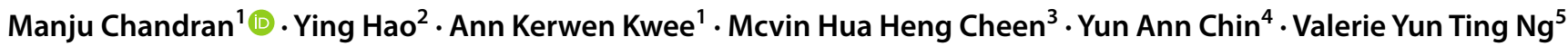

1 Osteoporosis and Bone Metabolism Unit, Department of Endocrinology, Singapore General Hospital, Singapore, Singapore

2 National Public Health and Epidemiology Unit, National Centre for Infectious Diseases, Singapore, Singapore

3 Danone Trading Medical B.V, Hoofddorp, The Netherlands
4 Department of Endocrinology, Singapore General Hospital, Singapore, Singapore

5 Department of Pharmacy, Singapore General Hospital, Singapore, Singapore 